\title{
Letter to Editor: Collateral damage: impact of COVID-19 on stroke care in three downtown Los Angeles Community Hospitals
}

\author{
Diana Escobar $^{1} \cdot$ Cara Beth Rogers $^{1} \cdot$ Ian Christensen $^{1} \cdot$ Michael Jordan $^{1} \cdot$ Antonio Liu $^{1}$ (D)
}

Received: 22 January 2021 / Accepted: 26 January 2021 / Published online: 2 March 2021

(c) Belgian Neurological Society 2021

We read the paper by Sharma et al. published on 31 July 2020 in Stroke and Vascular Neurology with great interest [1]. Their study, conducted at several tertiary/academic hospitals described a 31.4\% decrease in stroke/TIA presentations and a $46.1 \%$ decrease in ED stroke alerts between 23 March and 19 April 2020 as compared to the year before. They concluded that much of this decrease could be attributed to increased reluctance to seek medical care due to concern of contracting COVID-19. In their discussion, the authors questioned whether their data were generalizable to the rest of the United States.

To help answer that query, we are reporting stroke/TIA presentations and ED stroke alert numbers from three certified stroke centers (one comprehensive, two primary) in community hospital settings in Los Angeles. These facilities have a total of 1158 hospital beds and serve a combined population of about 2.5 million people in non-overlapping catchment areas. A retrospective analysis was performed for these three hospitals from Jan 2020 to July 2020. Similar to the findings in the study conducted by Sharma et al., we found a significant decrease in stroke/TIA presentation in the months of March, April, and May, with the greatest decline in the month of April 2020, as compared to the same period in 2019 (Table 1).

During this 3-month period, there was a $25 \%$ decrease in stroke/TIA presentations (222 vs 296) as compared to the year before, and a $43 \%$ decline in ED stroke alerts (208 vs 362). This result is consistent with that found by Sharma et al. Interestingly, the degree to which these metrics declined in our population does not differ significantly from those described by Sharma et al. Given that our patient population is approximately $70-80 \%$ Hispanic, this suggests that the pandemic's deterrent effect on stroke presentation may be consistent regardless of cultural or geographic differences.

In addition, our analysis through the end of July further exhibits a substantial recovery to pre-pandemic levels with similar levels of stroke/TIA presentations and stroke alerts as in 2019 (Figs. 1, 2). This occurred despite the increasing incidence of COVID-19 throughout that time period in the Los Angeles area [2]. This could be due to increased familiarity with the COVID-19 virus leading to increased risk tolerance among our population [3]. This occurred in the absence of any significant public health intervention encouraging people to access medical care.

It will be interesting to see how the trend continues as COVID-19 cases continue to rise across the nation. Will

Table 1 Stroke, TIA and stroke alert activations during the 3 months of March, April and May, 2019 and 2020

\begin{tabular}{lccl}
\hline & $\begin{array}{l}\text { Mar, Apr and } \\
\text { May 2019 }\end{array}$ & $\begin{array}{l}\text { Mar, Apr and } \\
\text { May 2020 }\end{array}$ & $\begin{array}{l}\text { Percent } \\
\text { decrease }\end{array}$ \\
\hline Stroke & 201 & 166 & 17 \\
TIA & 95 & 56 & 41 \\
Stroke/TIA & 296 & 222 & 25 \\
Stroke alerts & 362 & 208 & 43 \\
\hline
\end{tabular}

Antonio Liu

anthonyseanliu@yahoo.com

1 Adventist Health White Memorial, Los Angeles, USA 


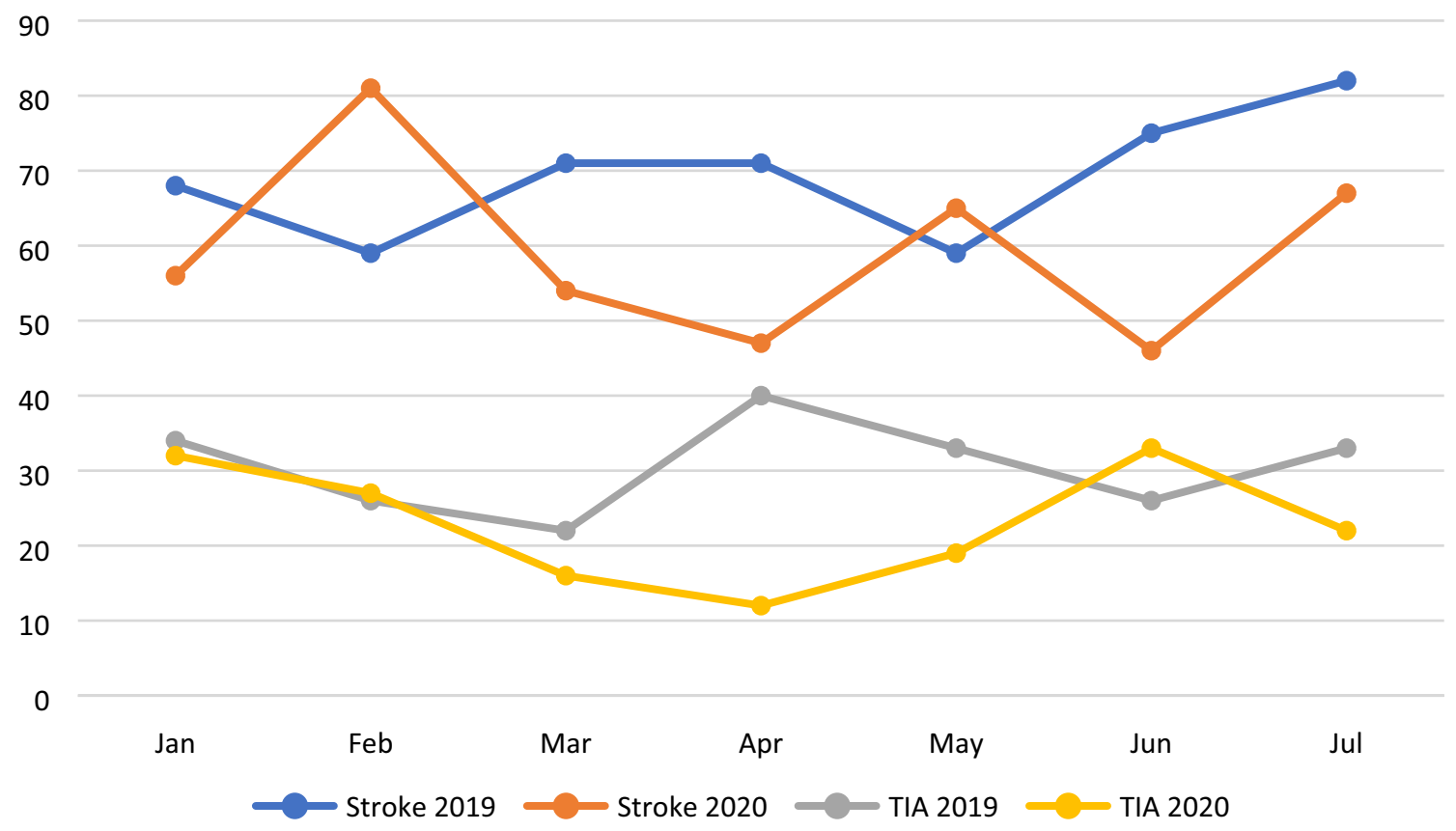

Fig. 1 Stroke and TIA encounter for Jan-Jul of 2019 and 2020

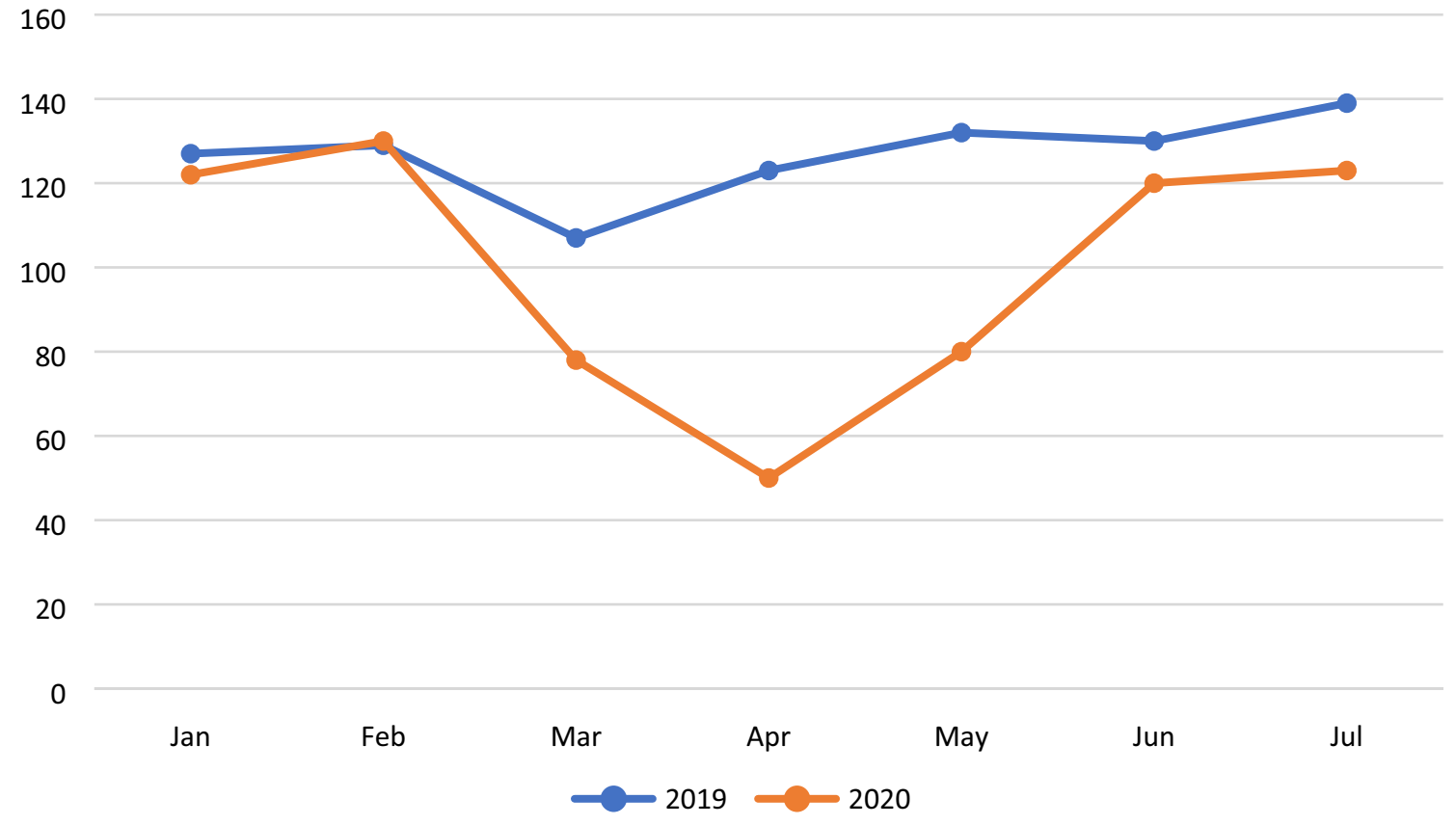

Fig. 2 Stroke alert activation Jan-Jul, 2019 and 2020 
visits to the emergency room for stroke continue to fluctuate proportionally, as seen during the initial months of the pandemic? Or will there be a return to pre-pandemic levels, such as those in July 2020, as people become more complacent about the pandemic or more comfortable with the precautions implemented at their local hospitals? The future trajectory of the pandemic is far from clear and it will remain important to track the trends in emergency care for stroke and TIA to assess for the possible need for public health interventions to ensure optimal care for stroke patients.

\section{Compliance with ethical standards}

Conflict of interest There is no conflict of interest of any kind to declare from any authors. There is no financial interest involved in this article.

Ethical approval This article does not contain any studies with human participants or animals performed by any of the authors.

Informed consent For this type of purely observational report, consent is not required.

\section{References}

1. Sharma M, Lioutas V, Madsen T et al (2020) Decline in stroke alerts and hospitalisations during the COVID-19 pandemic. Stroke Vasc Neurol. https://doi.org/10.1136/svn-2020-000441

2. LA County Daily COVID-19 Data_LA County Department of Public Health, http://publichealth.lacounty.gov/media/coronaviru s/data/index.htm.

3. Camacho-Rivera M et al (2020) Heterogeneity in trust of cancer information among Hispanic adults in the United States: an analysis of the health information national trends survey. Cancer Epidemiol BiomarkPrev. https://doi.org/10.1158/1055-9965. EPI-19-1375

Publisher's Note Springer Nature remains neutral with regard to jurisdictional claims in published maps and institutional affiliations. 